\title{
Preparation of Spherical and Porous Chitosan Particles by Suspension Evaporation with $\mathrm{O} / \mathrm{W} / \mathrm{O}$ Multiple Emulsions
}

\author{
Tsuneyasu Adachi, Jun-ichi Ida, Masa-aki WaKita, Masanori Hashimoto, \\ Hirotaka Ihara, ${ }^{*}$ and Chuichi Hirayama* \\ Kurita Water Industries Ltd., 7-1 Wakamiya Morinosato, \\ Atsugi, Kanagawa 243-0124, Japan \\ * Department of Applied Chemistry \& Biochemistry, Kumamoto University, \\ 2-39-1 Kurokami, Kumamoto 860-8555, Japan
}

(Received June 11, 1998)

\begin{abstract}
Porous spherical particles of chitosan were prepared by conventional suspension evaporation with modification. Aqueous chitosan solution was dispersed in decahydronaphthalene as the suspension medium with a nonionic surfactant as the emulsifier. $\mathrm{O} / \mathrm{w} / \mathrm{o}$ multiple emulsions consisting of aqueous chitosan particles containing decahydronaphthalene and dispersed in decahydronaphthalene were obtained. Chitosan precipitated as insoluble spherical particles by the gradual removal of water with heating. Particle porosity was related to microemulsions formation, the cloud point of the emulsifier and chemical structure of the acid used.

KEY WORDS Chitosan / Spherical Particles / Multiple Emulsions / Microemulsions / Suspension

Evaporation / Liquid Chromatography /
\end{abstract}

Methods for producing porous and spherical particles from polymers, such as suspension evaporation, ${ }^{1-6}$ wet coagulation, ${ }^{7}$ phase separation, ${ }^{8}$ and freeze-drying, ${ }^{9}$ are also used to obtain carrier particles for liquid chromatography and bioreactors. Suspension evaporation was developed by Motozato et $a l^{1,2}$ and has been applied to various polymers by Hirayama and Ihara. ${ }^{3-6}$ In this method, hydrophobic polymers dissolved in waterinsoluble solvents such as chloroform are dispersed in aqueous solution and polymers precipitate as solidified spherical particles by the gradual removal of the solvent. Reticulation of particles for creating pores is conducted by adding a poor polymer solvent as diluent to the suspension, which is removed after evaporation. Porosity and pore size can thus be easily controlled by the kind and amount of diluent. ${ }^{10-12}$

A modified suspension evaporation method was established in the present study to obtain porous and spherical particles from chitosan, a derivative of chitin, a natural polysaccharide. Chitosan should prove useful for chemical modification owing to its numerous reactive primary amino groups. Though conventional suspension evaporation is applied to water-insoluble polymers to produce $\mathrm{o} / \mathrm{w}$ emulsions (suspension), ${ }^{1-6}$ chitosan is a water-soluble polymer also useful for w/o emulsion preparation and was used in this study. The method for modified suspension evaporation in this study was based on the formation of $\mathrm{o} / \mathrm{w} / \mathrm{o}$ multiple emulsions produced by suspending aqueous chitosan o/w emulsions containing a hydrophobic diluent in a hydrophobic medium. In the conventional preparation of $\mathrm{o} / \mathrm{w} / \mathrm{o}$ multiple emulsions, two different surfactants are used ${ }^{13}$ and chitosan microspheres have recently been prepared with multiple emulsions. ${ }^{14}$ Maintaining an o/w/o multiple emulsion is difficult since suspension evaporation involves gradual heating and solvent evaporation. Thus, only one surfactant, decahydronaphthalene, that serves as both suspension medium and diluent, was used. The porosity of chitosan particles produced in this manner is disccused.

\section{EXPERIMENTAL}

\section{Materials}

Chitosan was purchased from Dainichiseika Color \& Chemicals Mfg. Co., Ltd. Colloidal titration ${ }^{15}$ indicated the degree of deacetylation as $84 \%$. Intrinsic viscosity was $23 \mathrm{dlg}^{-1}$ in $0.2 \mathrm{M}$ acetic acid- $0.1 \mathrm{M}$ sodium acetate buffer solution.

Polyoxyethylene nonylphenol (Nonipol) was used as the nonionic surfactant, Nonipol 70 (HLB: 11.7), Nonipol 95 (HLB: 13.1, cloud point $52-58^{\circ} \mathrm{C}$ ), Nonipol 110 (HLB: 13.8 , cloud point $70-76^{\circ} \mathrm{C}$ ), Nonipol 130 (HLB: 14.5, cloud point 83-89 C), and Nonipol 200 (HLB: 16, cloud point $>100^{\circ} \mathrm{C}$ ) were purchased from Sanyo Chemical Industries, Ltd. Decahydronaphthalene (decalin, Katayama Chemical Industries Co., Ltd.), $N, N$-dimethylformamide (DMF, Kishida Chemical Co., Ltd.), and hexamethylenediisocyanate (Tokyo Kasei Kogyo Co., Ltd.) were used without further purification.

\section{Preparation of Chitosan Films}

Chitosan $(0.5 \mathrm{~g})$ was dissolved in $0.5 \mathrm{wt} \%$ acetic acid $(100 \mathrm{ml})$ and filtrated with a $3 \mathrm{G} 1$ glass filter to remove insoluble matter. Nonipol ( $2.5 \mathrm{~g})$ was dissolved in decalin $(400 \mathrm{ml})$ and chitosan solution $(33 \mathrm{ml})$ was added to decalin with stirring at $2000 \mathrm{rpm}$ for $5 \mathrm{~min}$. The emulsion of chitosan aqueous solution containing decalin precipitated at room temperature without stirring and was separated from decalin with a separating funnel. The emulsions $(10 \mathrm{ml})$ were cast on glass petri dishes $(90 \mathrm{~mm}$ i.d.) and dried at $80^{\circ} \mathrm{C}$. The films were sufficiently washed with acetone.

Chitosan cast films were also obtained as follows: Nonipol $130(200 \mathrm{~g})$ was dissolved in water $(800 \mathrm{ml})$ followed by the addition of chitosan (16 g) and acid (1.2 equimolar for each amino group of chitosan). Emulsions were obtained by the addition of decalin $(20 \mathrm{ml})$ to this system $(60 \mathrm{ml})$ with homogenizing and the emulsions $(10 \mathrm{~mL})$ were cast on glass petri dishes $(90 \mathrm{~mm}$ i.d.) and 
dried at $80^{\circ} \mathrm{C}$. The films were sufficiently washed with acetone.

\section{Preparation of Spherical Chitosan Particles}

Chitosan $(10.0 \mathrm{~g})$ was dissolved in water $(500 \mathrm{~mL})$ containing adipic acid $(8.8 \mathrm{~g})$ and filtrated with a $17 \mathrm{Gl}$ glass filter to remove insoluble matter. Decalin $(800 \mathrm{ml})$ containing Nonipol $130(10.0 \mathrm{~g})$ was introduced into a three-necked stainless-steel vessel $(170 \mathrm{~mm} \times 130 \mathrm{~mm}$ i.d. $)$ coated with Teflon. Chitosan solution $(50 \mathrm{ml})$ was added to one of decalin with stirring, using a four-blade paddle ( $70 \mathrm{~mm}$ i.d.) at $2000 \mathrm{rpm}$. The stirring and blowing of nitrogen gas onto the air-to-solution interface were done at $78^{\circ} \mathrm{C}$ for $2 \mathrm{~h}$ to remove vapor containing water. The precipitates were obtained using a test sieve of $45 \mu \mathrm{m}$ mesh and washed with acetone. The particles neutralized by ethanol solution containing $3 \mathrm{M} \mathrm{NaOH}$ were suspended in a DMF-acetone mixture and crosslinked by the addition of hexamethylenediisocyanate and washed with acetone.

\section{Measurements}

Particle shape and surface structure of the chitosan particles and films dried in vacuo were observed by a scanning electron microscope (JEOL JSM-T100).

Ion-exchange capacity of the particles was assessed by pH-titration using $3 \mathrm{ml}$ particles dispersed in $0.1 \mathrm{M} \mathrm{NaCl}$ $(70 \mathrm{ml})$ and $1 / 10 \mathrm{~N}-\mathrm{HCl}$.

The particles were packed into a glass column (100 $\mathrm{mm} \times 5 \mathrm{~mm}$ i.d.). Flow rate-pressure drop was evaluated based on increase in the flow rate of water as the mobile phase.

\section{RESULTS AND DISCUSSION}

\section{Formation of $O / W$ Emulsions and Effects on Macro- reticulation}

A nonionic surfactant was used to stabilize o/w emulsions of chitosan aqueous solution containing decalin. The emulsions were dispersed in decalin solution by stirring. Chitosan, an emulsifier, and decalin were mixed by sharing stress with stirring to produce $\mathrm{o} / \mathrm{W}$ emulsions from chitosan aqueous solution containing decalin. After being stirred, the emulsions were phase-separated from decalin as the suspension medium. Each phase-separated chitosan emulsion contained nonionic surfactant at $c a$. $85 \%$.

Figure 1 shows typical SEM micrographs of chitosan films prepared from chitosan o/w emulsions. A SEM micrograph of film prepared without an emulsifier is shown in Figure 2. The film was transparent and nonporous. Films prepared by $\mathrm{o} / \mathrm{w}$ emulsions were opaque and porous. Decalin in the emulsions is thus shown to efficiently function as diluent for macroreticulation. The removal of decalin resulted in pore formation. As shown in Figure 1, pore-size distribution was affected by cloud points of the surfactants. A surfactant with cloud point near the sphering temperature produced homogeneous pores on the film surface. It would thus appear that pore-size distribution may bear some relation to the particle size of decalin in an emulsion. The cloud point of a surfactant and temperature may possibly be involved in minimizing decalin particle size in an
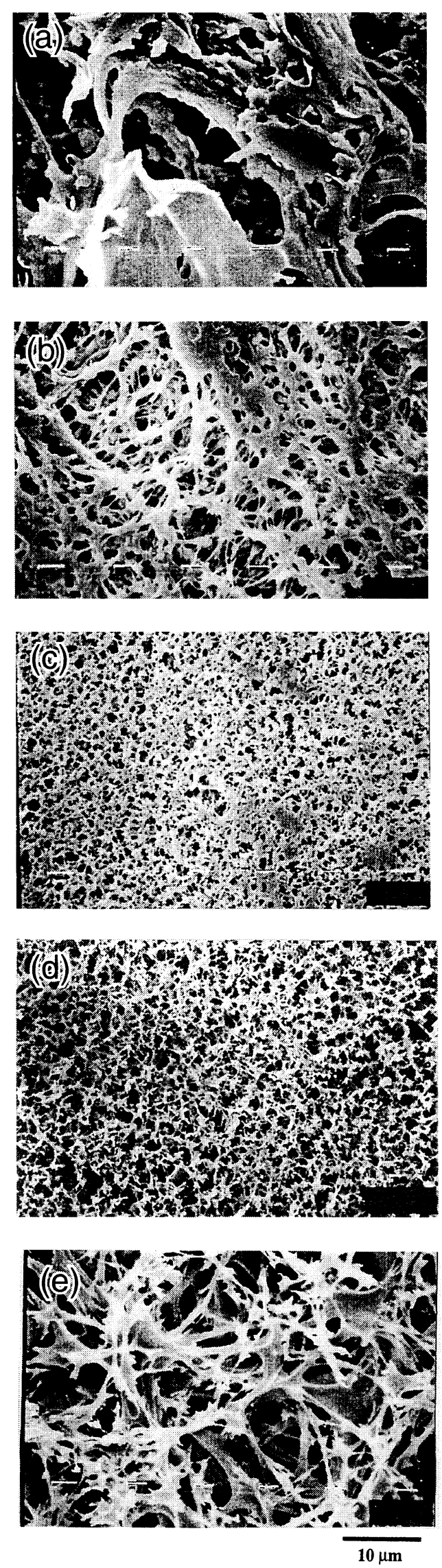

Figure 1. SEM micrographs of chitosan films prepared from $o / w$ chitosan emulsions containing decalin and different emulsifiers: (a) Nonipol 70 (HLB 11.7); (b) Nonipol 95 (HLB 13.1, cloud point $52-58^{\circ} \mathrm{C}$ ); (c) Nonipol 110 (HLB 13.8 , cloud point $70-76^{\circ} \mathrm{C}$ ); (d) Nonipol 130 (HLB 14.5, cloud point 83-89 ${ }^{\circ}$ ); (e) Nonipol 200 (HLB 16 , cloud point $>100^{\circ} \mathrm{C}$ ). 


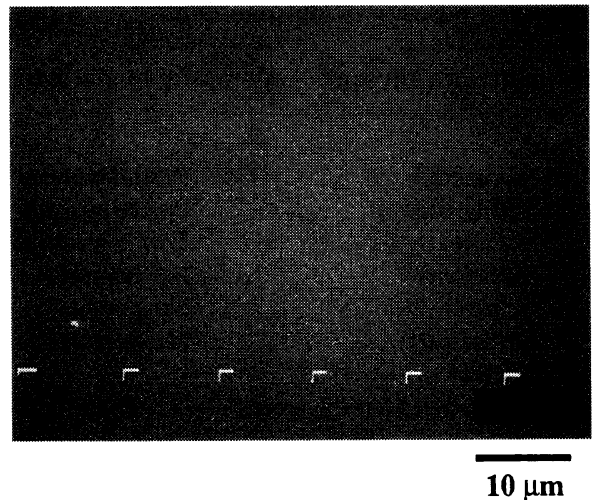

Figure 2. SEM micrographs of chitosan film prepared without an emulsifier.
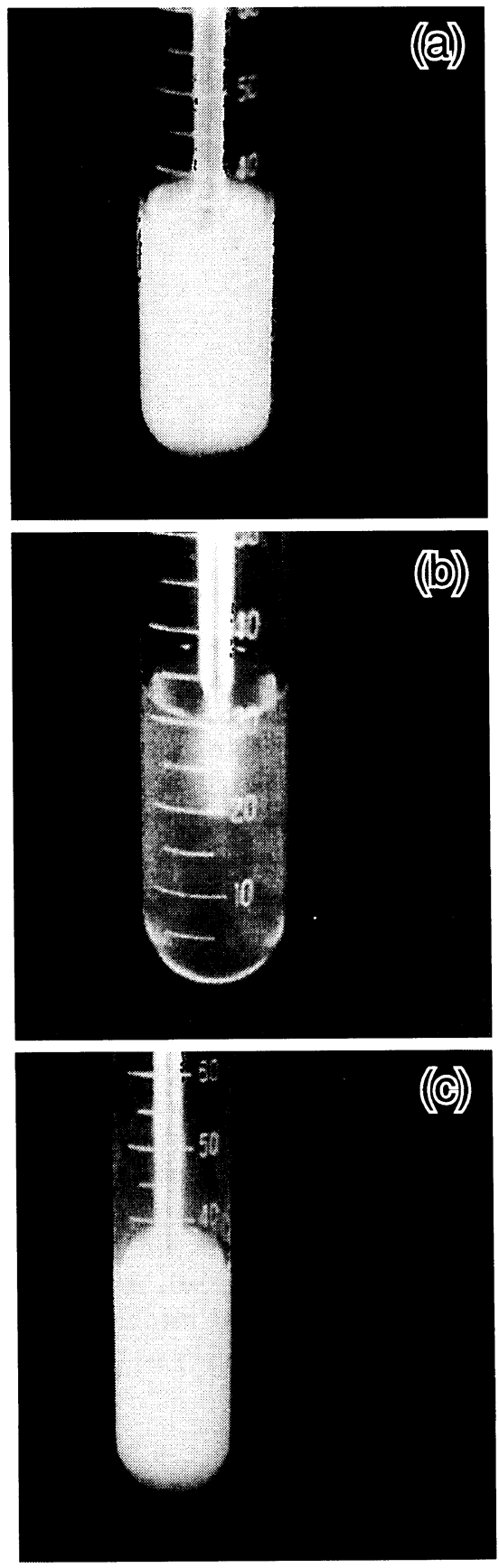

Figure 3. Photographs of $\mathrm{o} / \mathrm{w}$ emulsion prepared with chitosan aqueous solution, decalin and Nonipol 110 (HLB 13.8, cloud point $70-76^{\circ} \mathrm{C}$ ) at different temperatures: (a) room temperature; (b) $60-70^{\circ} \mathrm{C}$; (c) $85^{\circ} \mathrm{C}$.
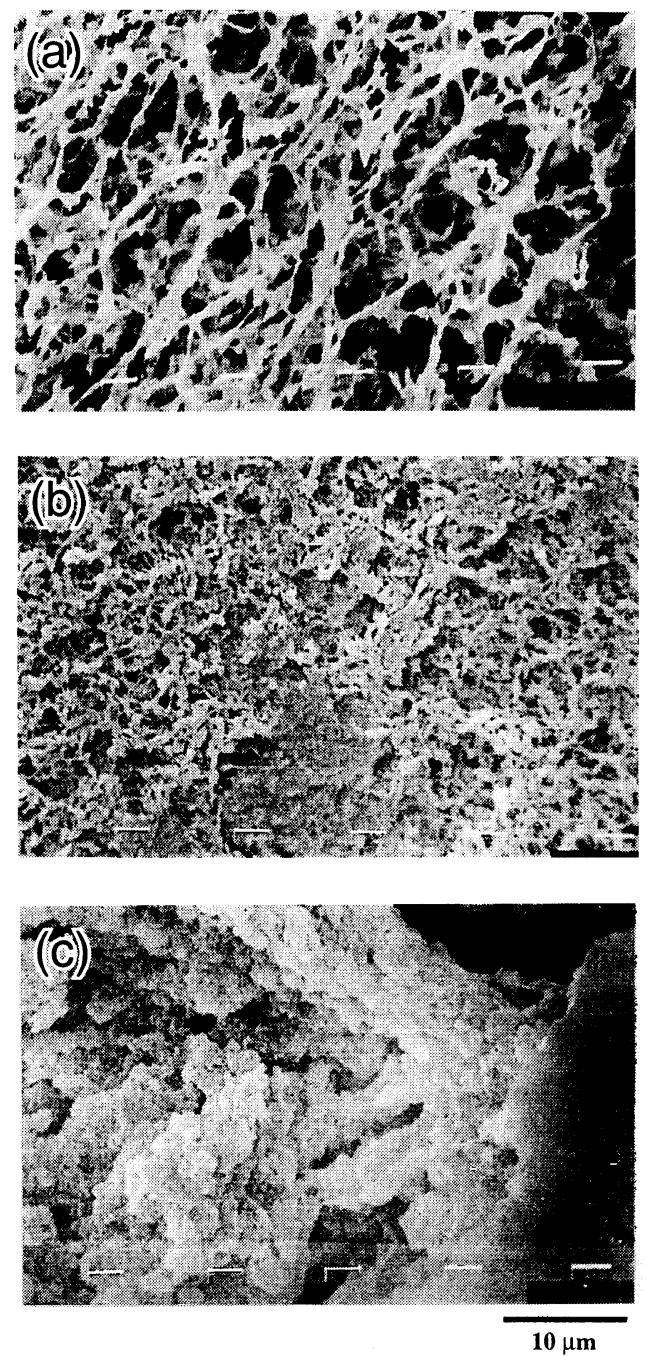

Figure 4. SEM micrographs of chitosan films prepared from $o / w$ chitosan emulsions. Chitosan aqueous solutions were obtained using the following acids: (a) adipic acid; (b) acetic acid; (c) hydrochloric acid.

emulsion

\section{Temperature Dependency of $\mathrm{O} / \mathrm{W}$ Emulsions}

Using two nonionic surfactants with cloud points $\left(T_{\mathrm{c}}\right)$ at $70-76^{\circ} \mathrm{C}$ and $83-89^{\circ} \mathrm{C}, \mathrm{o} / \mathrm{w}$ emulsions of chitosan aqueous solution containing decalin were colorimetrically compared while increasing temperature.

The emulsion obtained in either case was milk-white at room temperature and turned colorless and transparent at temperatures somewhat below $T_{\mathrm{c}} \mathrm{s}$, and the milk-white color disappeared with increase in temperature above $T_{\mathrm{c}} \mathrm{s}$, as shown in the Figure 3. Transparent emulsions are often called microemulsions ${ }^{16.17}$ and decalin may possibly be dispersed as nano particles. That chitosan microemulsions were produced near $T_{\mathrm{c}}$ and homogeneous pores were formed on chitosan film (Figure 1) when using a surfactant having $T_{\mathrm{c}}$ similar to the sphering temperature indicates increase in temperature to induce the formation of microemulsions containing phase-separated decalin as homogeneous nano particles. $\mathrm{O} / \mathrm{w}$ emulsions without chitosan showed no change in color or transparency regardless of temperature. It thus follows that chitosan as well as surfactants stabilizes decalic particles in $\mathrm{o} / \mathrm{w}$ emulsions. 
Table I. Evaluation of acid capacity for inducing chitosan solubility and porosity of chitosan film

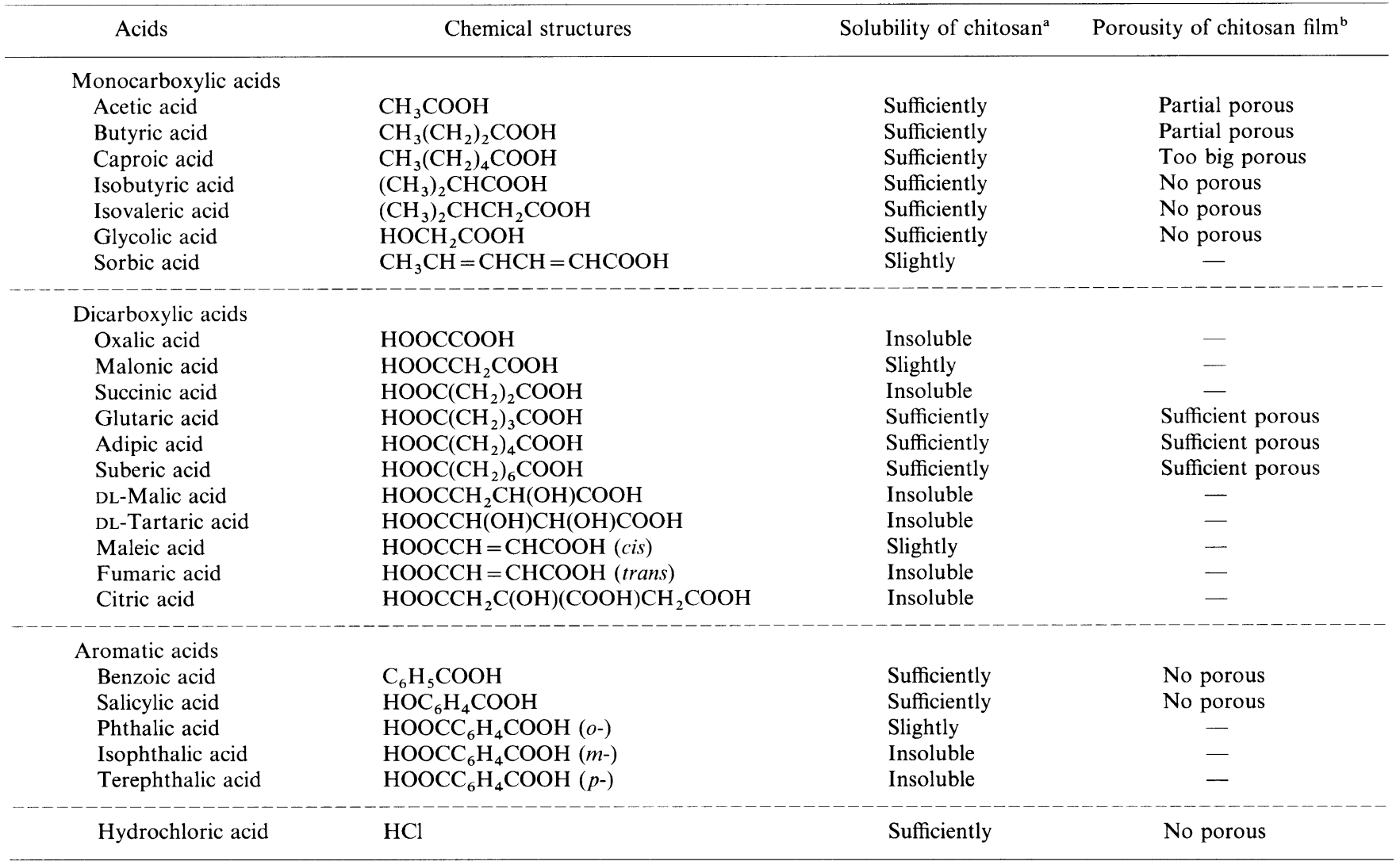

${ }^{a}$ Solubility of chitosan judged visually for $2 \mathrm{wt} \%$ chitosan aqueous solutions with acids (1.2 equimolar for amino group of chitosan). ${ }^{\mathrm{b}}$ Porosity of film judged visually for SEM micrographs of films prepared from chitosan $\mathrm{o} / \mathrm{w}$ emulsions.

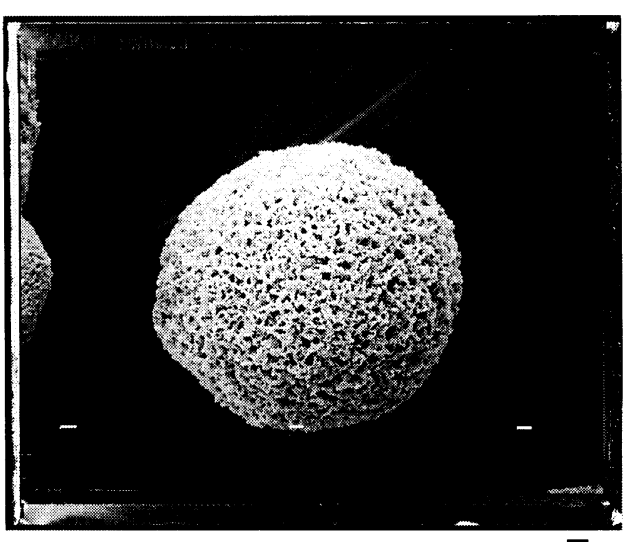

$10 \overline{\mu m}$

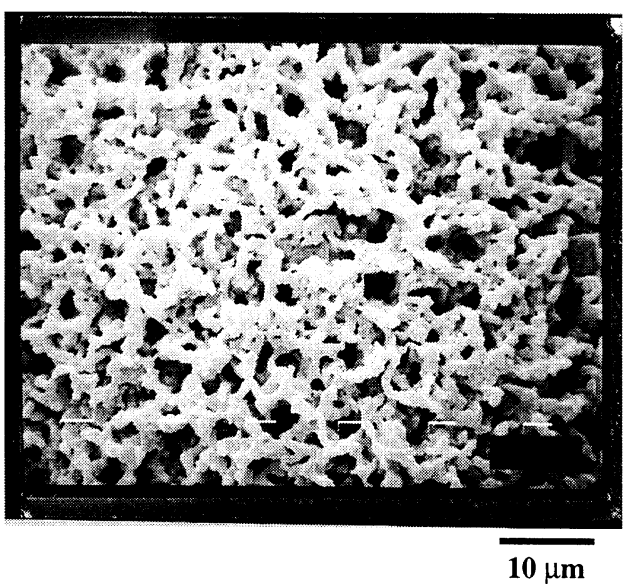

Figure 5. SEM micrographs of chitosan particles prepared by suspension evaporation.

\section{Effects of Acid on Macroreticulation}

Chitosan was dissolved in acidic aqueous solution and thus its interactions with surfactants should be affected by the acid used. The acids used, solubility of chitosan in aqueous solution and morphological features of pores produced on chitosan film are indicated in Table I. Figure 4 shows typical SEM micrographs of chitosan film prepared using adipic, acetic, and hydrochloric acids. Adipic, glutaric and suberic acids as dicarboxylic acids gave good chitosan solubility. With these dicarboxylic acids, homogeneous macropores were produced on the film surface. Macroreticulation failed to occur in the case of some monocarboxylic, aromatic, and hydrochloric acids although increase in the solubility was observed. Accordingly, the dicarboxylic acids may modify chitosan to stabilize decalin particles in o/w emulsions by changing the hydrophilic and hydrophobic features of chitosan. The initial concentration of chitosan was noted to affect film reticulation. Partially porous film (Figure 4 (b)) was produced with $2 \mathrm{wt} \%$ chitosan solution prepared using acetic acid, while film obtained using $0.5 \mathrm{wt} \%$ chitosan solution prepared with acetic acid was completely porous (Figure 1 (c)).

\section{Preparation of Spherical Chitosan Particles}

Figure 5 shows SEM micrographs of chitosan particles prepared by suspension evaporation and all are completely spherical. Particle diameter and pore size on the surface were $0.2 \mathrm{~mm}$ and $5 \mu \mathrm{m}$, respectively, on the average. Macropores were present within the particles. Weight per $1 \mathrm{ml}$ chitosan particles dried in vacuo was 


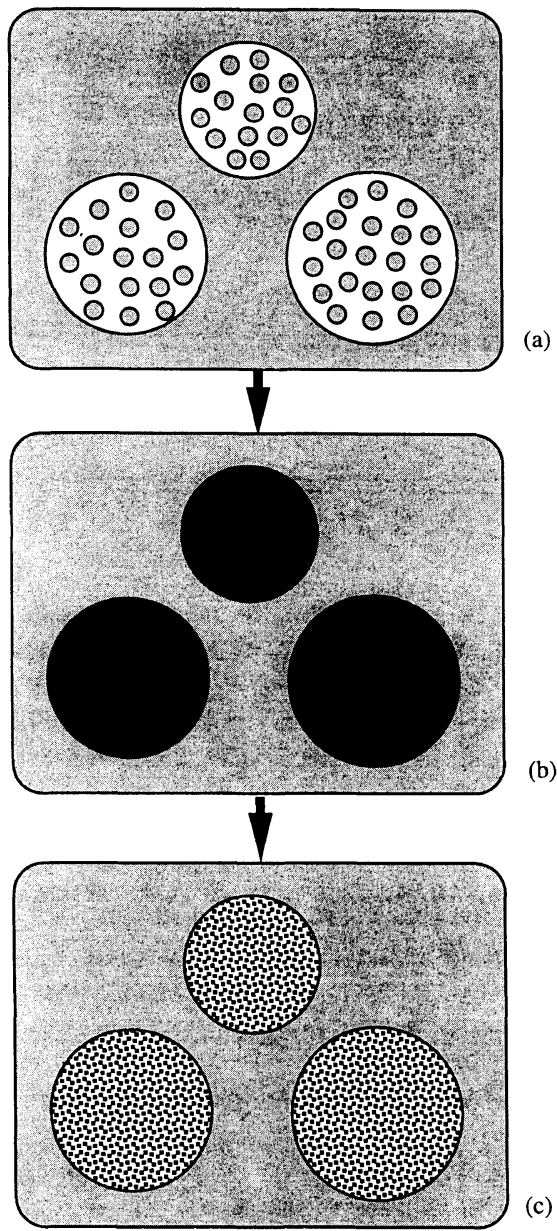

Figure 6. Mechanism for macroreticulation of chitosan particles. (a) Chitosan aqueous solution was dispersed in decalin containing an emulsifier to produce an o/w/o multiple emulsion. (b) $\mathrm{O} / \mathrm{w}$ chitosan emulsions became microemulsions with increase in temperature. (c) Chitosan precipitated as insoluble spherial particles by the gradual removal of water and macropores were created by removal of decalin.

$0.1 \mathrm{~g}$. Ion-exchange capacity and maximum flow-rate in

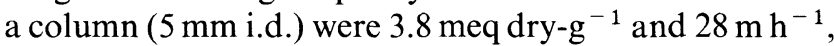
respectively. Chitosan particles prepared somewhat below $T_{\mathrm{c}}\left(78^{\circ} \mathrm{C}\right)$ had homogenous macropores within the particles, but partially porous chitosan particles were produced at nearly $T_{\mathrm{c}}\left(83^{\circ} \mathrm{C}\right)$. Microemulsion formation is thus shown to effectively bring about macroreticulation through suspension evaporation.

Figure 6 presents the mechanism proposed for the macroreticulation of chitosan particles. In suspension evaporation, water, a good solvent for chitosan, is evaporated from the chitosan emulsion which then precipitated as spherical and solidified particles. Decalin is effective for macroreticulation and also functions as a suspension medium for the emulsion. $\mathrm{O} / \mathrm{w}$ emulsions made using chitosan aqueous solution containing decalin become microemulsions with increase in temperature and are essential for homogeneous pores.

\section{CONCLUSIONS}

Spherical and porous chitosan particles were prepared by suspension evaporation with modification. Conventional suspension evaporation has been applied to hydrophobic polymers, but chitosan is a very hydrophilic polymer. The chitosan emulsions in this study were prepared using adipic acid aqueous solution and decalin and were dispersed in decalin. The chitosan particles were completely spherical and had macroreticulate pores. Some were hydrophilic and possessed primary amino groups. Fairly high molecular weight polymers should thus be capable of entering chitosan particles without non-specific adsorption. Accordingly, chitosan particles should prove useful as adsorbents for anionic bio-related polymers such as proteins, endotoxins and nucleic acids or carriers for cell cultures.

\section{REFERENCES}

1. Y. Motozato and C. Hirayama, Nippon Kagaku Kaishi, 1087 (1972).

2. Y. Motozato and C. Hirayama, J. Chromatogr., 298, 499 (1984).

3. Y. Motozato, H. Ihara, T. Tomoda, and C. Hirayama, $J$. Chromatogr., 355, 434 (1986).

4. C. Hirayama, H. Ihara, M. Shiba, M. Nakamura, Y. Motozato, and T. Kunitake, J. Chromatogr., 409, 175 (1987).

5. H. Ihara, T. Yoshinaga, Y. Motozato, and C. Hirayama, Polym. J., 17, 1301 (1985).

6. H. Ihara, T. Yoshinaga, and C. Hirayama, J. Chromatogr., 362, 197 (1986).

7. H. Seo and Y. Kinemura, Proc. 4th Int. Conf. on Chitin and Chitosan, 1988, p 585.

8. K. Okuma and H. Yoshidome, Polyfile, 28, 48 (1991).

9. S. Hjerten, Biochem. Biophys. Acta., 79, 393 (1964).

10. H. Ihara, H. Furusawa, X. Li, and C. Hirayama, J. Appl. Polym. Sci., 41, 347 (1991).

11. Y. Motozato, H. Ihara, T. Tomoda, and C. Hirayama, Nippon Kagaku Kaishi, 1187 (1986).

12. Y. Motozato, H. Ihara, M. Nakamura, M. Shiba, and C. Hirayama, Nippon Kagaku Kaishi, 61 (1988).

13. S. Matsumoto, Y. Kita, and D. Yonezawa, J. Colloid. Interface Sci., 57, 353 (1976).

14. F. Pavanetto, P. Perugini, M. Modene, and I. Genta, J. Microencapsulation, 13, 679 (1996).

15. H. Teruyama, J. Polym. Sci., 8, 243 (1952).

16. J. H. Schulman, W. Stoeckenius, and L. M. Prince, J. Phys. Chem., 63, 1677 (1959).

17. K. Shinoda and H. Kunieda, J. Colloid Interface Sci., 42, 381 (1973). 